\title{
Runge-Kutta Methods of Higher Order for Solving Stiff Problems
}

Mohammed Mahmood Salih

mohammed.salihcs@uomosul.edu.iq

College of Computer Sciences and Mathematics

University of Mosul

Received on: 23/10/2004

\section{Basheer Mohammed Salih}

College of Education

University of Mosul

Accepted on: 05/04/2005

\begin{abstract}
Our purpose in this research is the development of higher order Runge-Kutta methods for solving stiff systems. We have developed methods of order five, six, and seven. We studied their stability Region and applications for solving stiff systems. Then we developed the corresponding implicit forms of these methods and we analyzed their stability and implementation for solving stiff systems.
\end{abstract}

Keywords: Runge-Kutta methods of higher order, ordinary differential equations, stiff problems.

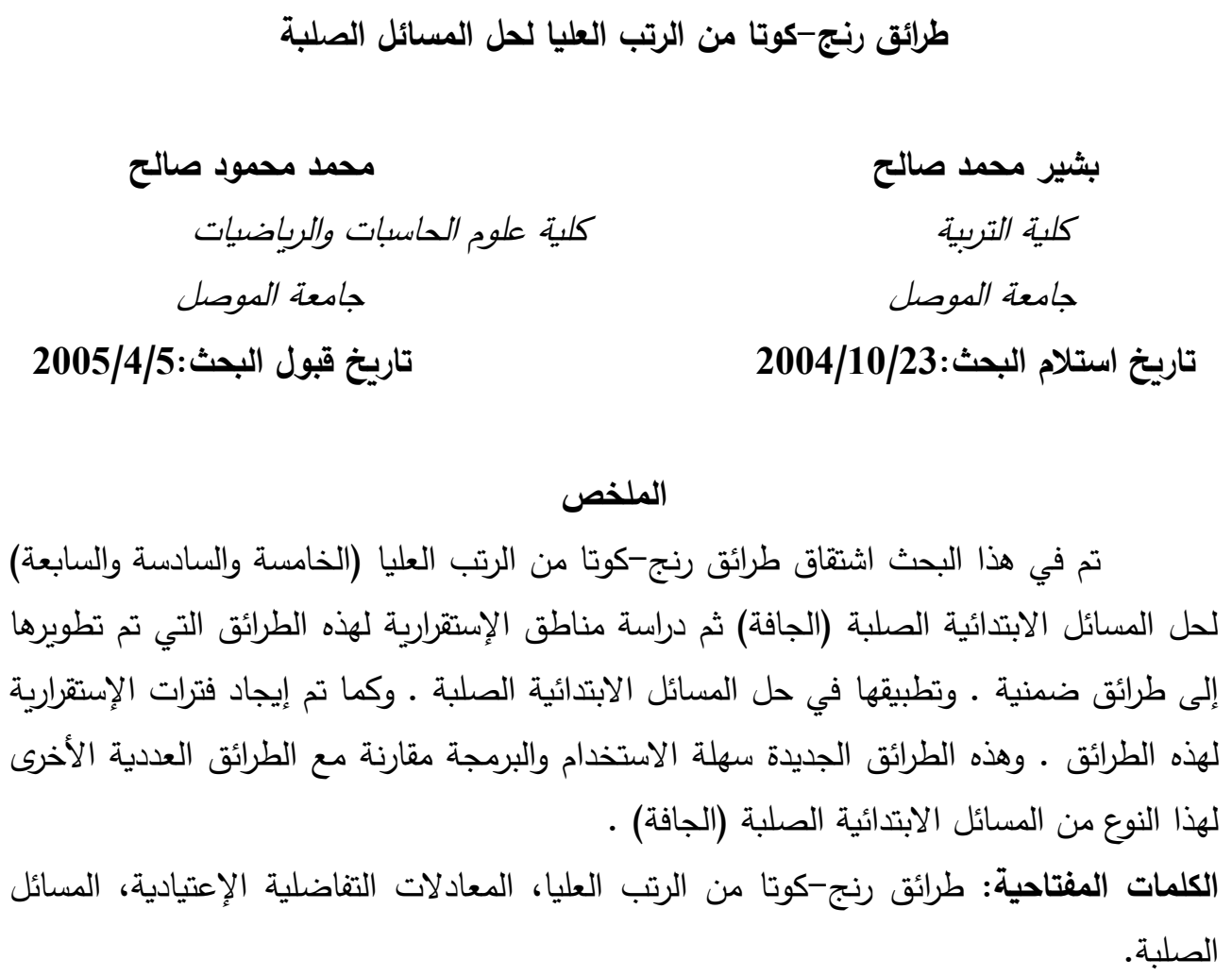


1. المقدمة:

ظهرت المسائل الصلبة منذ نصف قرن ،ومضت عليها بضع سنين من الإهمال حتى قال

العالم G.Dahquist في نحو 1960 " أصبح كل واحد مدركاً أن العالم مليء بالمسائل الصلبة ". أستخدمت مسائل القيم الإبتدائية عند دراسة حركة النوابض ذات الصلابة المختلفة ومنها أشتقت

[2] المسألة إسمها. [2]

كان أول ظهور لمصطلح الصلابة في بحث لـ Hirschfelder و في مسألة في علم الكيمياء الحركية ،إذ قاما بإقتراح أول مجموعة من صيخ التكاملات العددية

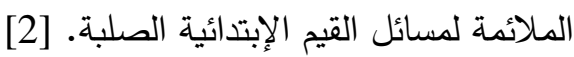
ثم طور Cash سنة 1975 الطرائق ذات الخطوة الواحدة فجاءت مشابهة في التصميم لطرائق رنج-كوتا للتكامل العددي الفعالة للأنظمة الجافة وغير الجافة من المعادلات التفاضلية من

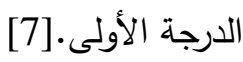

كما طور Cash سنة 1983 حساباً ملائماً للتكامل العددي للأنظمة الجافة يضم صيغ

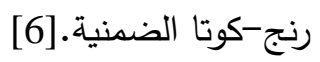
وفي السنوات الأخيرة أبتكرت طرائق عددية للمعادلات التفاضلية الصلبة وتم تطبيق قسم النمات منها بنجاح على أنواع معينة من المعادلات التفاضلية الصلبة.

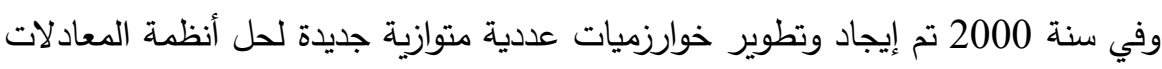
التفاضلية الإعتيادية الجافة كما تم إثتقاق طرائق رنج-كوتا الضمنية الجزئية التوازي.ـ10]

$$
\text { 2. طرائق رنج-كوتا الصريحة من الرتب العليا: }
$$

تعد طرائق رنج-كوتا الصريحة من الرتب العليا من الطرائق المهمة في حل أنظمة التهاب المعادلات التفاضلية الصلبة إذ تعطينا نتائج قريبة جداً إلى النتائج الحقيقية. ولقد إهتم العلماء والباحثون في إيجاد طرائق رنج-كوتا الصريحة من الرتب العليا للحيلولة دون الحصول على نتائج غير صحيحة بتاتاً.

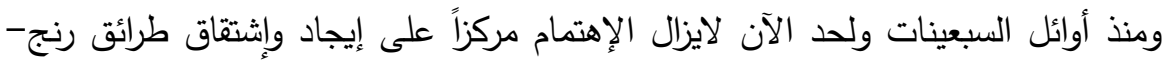
كوتا الصريحة من الرتب العليا وتطويرها بحيث تعطينا دقة في النتائج وسهولة في الإستخدام. سنتطرق الآن إلى طرائق رنج-كوتا الصريحة من الرتب العليا التي تم التوصل إليها. 
2.1 طريقة رنج كوتا الصريحة من الرتبة الخامسة:[3] إن صيغة رنج-كوتا الصريحة من الرتبة الخامسة تكون بالثكل الآتي :

$\mathrm{y}_{\mathrm{n}+1}=\mathrm{y}_{\mathrm{n}}+\frac{\mathrm{h}}{48}\left(8 \mathrm{k}_{1}+20 \mathrm{k}_{2}+12 \mathrm{k}_{3}+6 \mathrm{k}_{4}+\mathrm{k}_{5}+\mathrm{k}_{6}\right)$

$\mathrm{k}_{1}=\mathrm{f}\left(\mathrm{x}_{\mathrm{n}}, \mathrm{y}_{\mathrm{n}}\right)$

$\mathrm{k}_{2}=\mathrm{f}\left(\mathrm{x}_{\mathrm{n}}+\frac{\mathrm{h}}{2}, \mathrm{y}_{\mathrm{n}}+\frac{1}{2} \mathrm{hk}_{1}\right)$

$\mathrm{k}_{3}=\mathrm{f}\left(\mathrm{x}_{\mathrm{n}}+\frac{\mathrm{h}}{2}, \mathrm{y}_{\mathrm{n}}+\frac{1}{2} \mathrm{hk}_{2}\right)$

$\mathrm{k}_{4}=\mathrm{f}\left(\mathrm{x}_{\mathrm{n}}+\mathrm{h}, \mathrm{y}_{\mathrm{n}}+\mathrm{hk}_{3}\right)$

$\mathrm{k}_{5}=\mathrm{f}\left(\mathrm{x}_{\mathrm{n}}+\mathrm{h}, \mathrm{y}_{\mathrm{n}}+\mathrm{hk}_{4}\right)$

$\mathrm{k}_{6}=\mathrm{f}\left(\mathrm{x}_{\mathrm{n}}+\mathrm{h}, \mathrm{y}_{\mathrm{n}}+\mathrm{hk}_{5}\right)$

فترة إستقرارية هذه الطريقة (3.2,0-) [ انظر الثكل (1.1) ] .

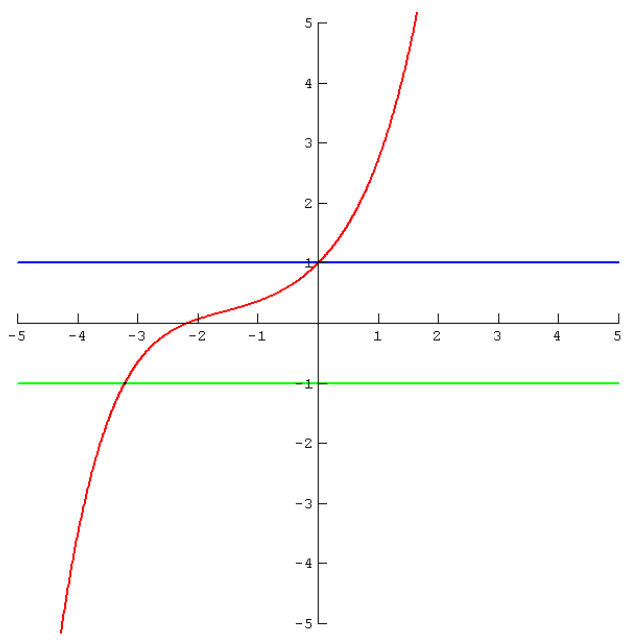

الشكل (1.1) فترة إستقرارية طريقة رنج-كوتا الصريحة من الرتبة الخامسة

2.2 طريقة رنج-كوتا الصريحة من الرتبة السادةة: [3] تكون صيغة رنج-كوتا الصريحة من الرتبة السادسة بالثكل الآتي : 
$\mathrm{y}_{\mathrm{n}+1}=\mathrm{y}_{\mathrm{n}}+\frac{\mathrm{h}}{96}\left(16 \mathrm{k}_{1}+40 \mathrm{k}_{2}+24 \mathrm{k}_{3}+12 \mathrm{k}_{4}+2 \mathrm{k}_{5}+\mathrm{k}_{6}+\mathrm{k}_{7}\right)$

$\mathrm{k}_{1}=\mathrm{f}\left(\mathrm{x}_{\mathrm{n}}, \mathrm{y}_{\mathrm{n}}\right)$

$\mathrm{k}_{2}=\mathrm{f}\left(\mathrm{x}_{\mathrm{n}}+\frac{\mathrm{h}}{2}, \mathrm{y}_{\mathrm{n}}+\frac{1}{2} \mathrm{hk}_{1}\right)$

$\mathrm{k}_{3}=\mathrm{f}\left(\mathrm{x}_{\mathrm{n}}+\frac{\mathrm{h}}{2}, \mathrm{y}_{\mathrm{n}}+\frac{1}{2} \mathrm{hk}_{2}\right)$

$\mathrm{k}_{4}=\mathrm{f}\left(\mathrm{x}_{\mathrm{n}}+\mathrm{h}, \mathrm{y}_{\mathrm{n}}+\mathrm{hk}_{3}\right)$

$\mathrm{k}_{5}=\mathrm{f}\left(\mathrm{x}_{\mathrm{n}}+\mathrm{h}, \mathrm{y}_{\mathrm{n}}+\mathrm{hk}_{4}\right)$

$\mathrm{k}_{6}=\mathrm{f}\left(\mathrm{x}_{\mathrm{n}}+\mathrm{h}, \mathrm{y}_{\mathrm{n}}+\mathrm{hk}_{5}\right)$

$\mathrm{k}_{7}=\mathrm{f}\left(\mathrm{x}_{\mathrm{n}}+\mathrm{h}, \mathrm{y}_{\mathrm{n}}+\mathrm{hk}_{6}\right)$

فترة الإستقرارية لهذه الطريقة (3.6,0-) [ انظر الثكل (1.2) ] .

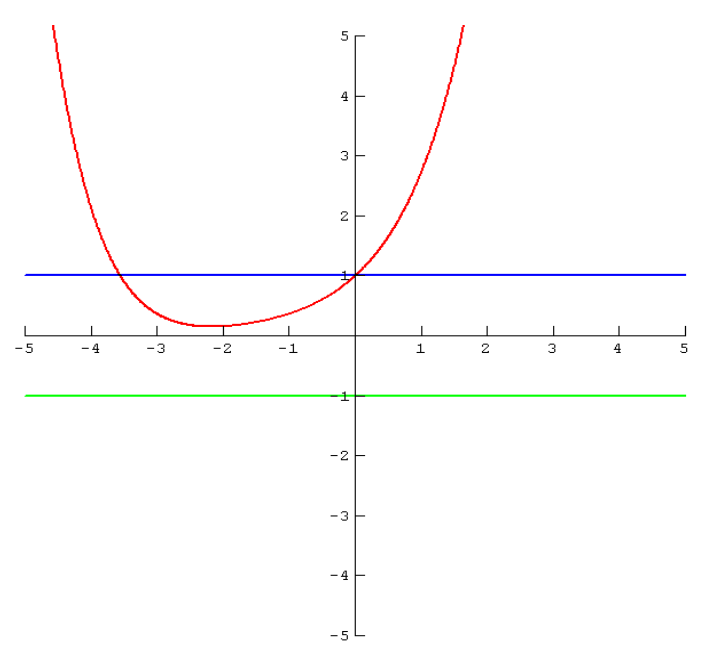

الثكل (1.2) فترة إستقرارية طريقة رنج-كوتا الصريحة من الرتبة السادسة

2.3 طريقة رنج-كوتا الصريحة من الرتبة السابعة: [3]

صيغة رنج-كوتا الصريحة من الرتبة السابعة تكون بالثكل الآتي:

$\mathrm{y}_{\mathrm{n}+1}=\mathrm{y}_{\mathrm{n}}+\frac{\mathrm{h}}{192}\left(32 \mathrm{k}_{1}+80 \mathrm{k}_{2}+48 \mathrm{k}_{3}+24 \mathrm{k}_{4}+4 \mathrm{k}_{5}+2 \mathrm{k}_{6}+\mathrm{k}_{7}+\mathrm{k}_{8}\right)$

حيث أن 


$$
\begin{aligned}
\mathrm{k}_{1} & =\mathrm{f}\left(\mathrm{x}_{\mathrm{n}}, \mathrm{y}_{\mathrm{n}}\right) \\
\mathrm{k}_{2} & =\mathrm{f}\left(\mathrm{x}_{\mathrm{n}}+\frac{\mathrm{h}}{2}, \mathrm{y}_{\mathrm{n}}+\frac{1}{2} \mathrm{hk}_{1}\right) \\
\mathrm{k}_{3} & =\mathrm{f}\left(\mathrm{x}_{\mathrm{n}}+\frac{\mathrm{h}}{2}, \mathrm{y}_{\mathrm{n}}+\frac{1}{2} \mathrm{hk}_{2}\right) \\
\mathrm{k}_{4} & =\mathrm{f}\left(\mathrm{x}_{\mathrm{n}}+\mathrm{h}, \mathrm{y}_{\mathrm{n}}+\mathrm{hk}_{3}\right) \\
\mathrm{k}_{5} & =\mathrm{f}\left(\mathrm{x}_{\mathrm{n}}+\mathrm{h}, \mathrm{y}_{\mathrm{n}}+\mathrm{hk}_{4}\right) \\
\mathrm{k}_{6} & =\mathrm{f}\left(\mathrm{x}_{\mathrm{n}}+\mathrm{h}, \mathrm{y}_{\mathrm{n}}+\mathrm{hk}_{5}\right) \\
\mathrm{k}_{7} & =\mathrm{f}\left(\mathrm{x}_{\mathrm{n}}+\mathrm{h}, \mathrm{y}_{\mathrm{n}}+\mathrm{hk}_{6}\right) \\
\mathrm{k}_{8} & =\mathrm{f}\left(\mathrm{x}_{\mathrm{n}}+\mathrm{h}, \mathrm{y}_{\mathrm{n}}+\mathrm{hk}_{7}\right)
\end{aligned}
$$

فترة الإستقرارية لهذه الطريقة (3.7,0-) [ انظر الثكل (1.3) ] .

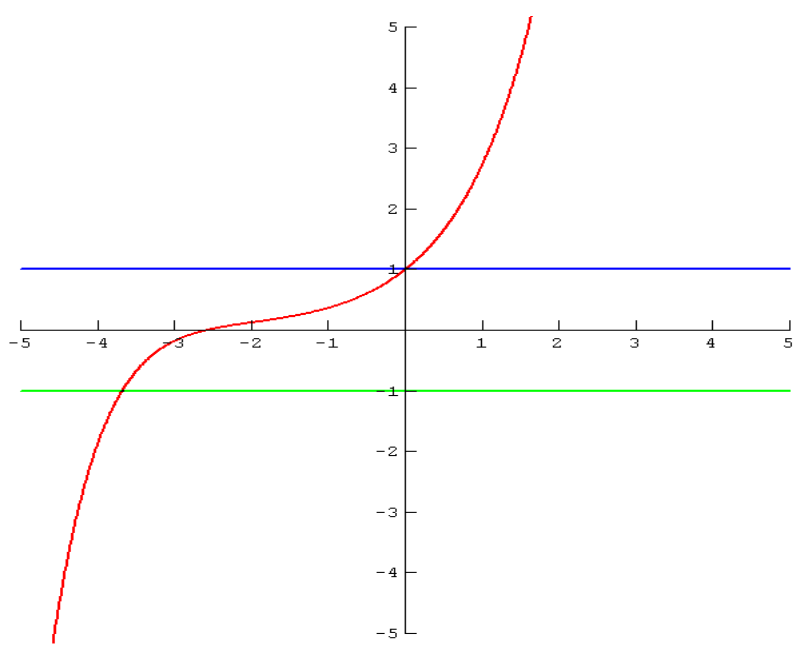

الثكل (1.3) فترة إستقرارية طريقة رنج-كوتا الصريحة من الرتبة السابعة

\section{4}

[2,1] : تأمل مسألة القيم الإبتدائية الآتية

$$
\mathrm{y}^{\prime}=\mathrm{f}(\mathrm{x}, \mathrm{y}), \mathrm{y}\left(\mathrm{x}_{0}\right)=\mathrm{y}_{0}, \mathrm{x} \in[\mathrm{a}, \mathrm{b}]
$$

إن الصيغة العامة لطريقة رنج-كوتا من المرحلة R تكون بالثكل الآتي : [2,1]

$$
\mathrm{y}_{\mathrm{n}+1}=\mathrm{y}_{\mathrm{n}}+\mathrm{h} \phi\left(\mathrm{x}_{\mathrm{n}}, \mathrm{y}_{\mathrm{n}}, \mathrm{h}\right)
$$

يقال أن الصيغة العامة لطريقة رنج-كوتا من المرحلة R أنها من الرتبة P إذا كان : [2,1]

$$
y\left(x_{n+1}\right)-y_{n+1}=O\left(h^{p+1}\right)
$$


بإستخدام الصيغة (2) على معادلة الفروقات نحصل على ما يأتي : [2,1]

$\mathrm{y}_{\mathrm{n}+1}=\mathrm{E}(\lambda \mathrm{h}) \mathrm{y}_{\mathrm{n}}$

وبإستخدام مسألة الإختبار نحصل على ما يأتي : [2,1)

$\mathrm{y}^{\prime}=\lambda \mathrm{y}, \mathrm{y}\left(\mathrm{x}_{0}\right)=\mathrm{y}_{0}$

وهكذا فإن :

$\mathrm{E}(\overline{\mathrm{h}})=\mathrm{r}_{1}=1+\overline{\mathrm{h}}+\frac{1}{2 !} \overline{\mathrm{h}}^{2}+\ldots \ldots \ldots \ldots \ldots+\frac{1}{\mathrm{p} !} \overline{\mathrm{h}}^{\mathrm{p}}+\mathrm{O}\left(\overline{\mathrm{h}}^{\mathrm{p}+1}\right)$

حيث أن R

وعليه تكون فترات الإستقرارية لطرائق رنج-كوتا من الرتب العليا كما في الجدول الآنس الآتي.

مع الأخذ بنظر الإعتبار أنه تم إيجاد هذه الفترات بإستخدام الرسم الآتي :

\begin{tabular}{|c|c|c|}
\hline RK & $r_{1}$ & فترة الإستقرارية \\
\hline 5 & $1+\overline{\mathrm{h}}+\frac{\overline{\mathrm{h}}^{2}}{2 !}+\frac{\overline{\mathrm{h}}^{3}}{3 !}+\frac{\overline{\mathrm{h}}^{4}}{4 !}+\frac{\overline{\mathrm{h}}^{5}}{5 !}$ & $(-3.2,0)$ \\
\hline 6 & $1+\overline{\mathrm{h}}+\frac{\overline{\mathrm{h}}^{2}}{2 !}+\frac{\overline{\mathrm{h}}^{3}}{3 !}+\frac{\overline{\mathrm{h}}^{4}}{4 !}+\frac{\overline{\mathrm{h}}^{5}}{5 !}+\frac{\overline{\mathrm{h}}^{6}}{6 !}$ & $(-3.6,0)$ \\
\hline 7 & $1+\overline{\mathrm{h}}+\frac{\overline{\mathrm{h}}^{2}}{2 !}+\frac{\overline{\mathrm{h}}^{3}}{3 !}+\frac{\overline{\mathrm{h}}^{4}}{4 !}+\frac{\overline{\mathrm{h}}^{5}}{5 !}+\frac{\overline{\mathrm{h}}^{6}}{6 !}+\frac{\overline{\mathrm{h}}^{7}}{7 !}$ & $(-3.7,0)$ \\
\hline
\end{tabular}

2.5 تطبيقات على المسائل الصلبة:

في هذه الفقرة سوف يتم تطبيق طرائق رنج-كوتا الصريحة من الرتب العليا على المسائل الصلبة مع بيان مدى كفاءة هذه الطرائق :

مثال (2.5.1) : (2.1)

حل نظام المعادلات التفاضلية الآتي :

$\mathrm{y}_{1}^{\prime}=\mathrm{y}_{2}, \mathrm{y}_{1}(\mathrm{O})=1$

$\mathrm{y}_{2}^{\prime}=-1001 \mathrm{y}_{2}-1000 \mathrm{y}_{1}, \mathrm{y}_{2}(0)=-1$

سوف نأخذ قيمة طول الخطوة h=0.002.

تثير طرائق رنج-كوتا الصريحة إلى مدى تأثير إستخدام طول الخطوة h ومدى كفاءة هذه

الطرائق مقارنة بالطرائق الاعتيادية . 
مثال (2.5.2)

حل نظام المعادلات التفاضلية الآتي :

$$
\begin{aligned}
& \mathrm{y}_{1}^{\prime}=600 \mathrm{y}_{1}{ }^{2}\left(\mathrm{y}_{2}-\mathrm{y}_{1}{ }^{3}\right), \mathrm{y}_{1}(0)=0.1 \\
& \mathrm{y}_{2}{ }^{\prime}=-200\left(\mathrm{y}_{2}-\mathrm{y}_{1}{ }^{3}\right)+2\left(1-\mathrm{y}_{2}\right), \mathrm{y}_{2}(0)=-0.1 \\
& \text { سوف نأخذ قيمة طول الخطوة h=0.001. }
\end{aligned}
$$

\begin{tabular}{|c|c|c|c|c|c|}
\hline \multicolumn{2}{|c|}{ قيم رنج- كوتا الصريحة من الرتبة } & \multicolumn{2}{|c|}{ القيم الحقيقية } & \multicolumn{2}{|c|}{ قيم الخطأ لرنج- كوتا الصريحة من } \\
\hline قيم & قيم & قيم & قيم & قيم الخطأ لـ y & قيم الخطأ لـ \\
\hline 1 & -1 & 1 & -1 & 0 & 0 \\
\hline 0.998 & -0.998 & 0.998 & -0.998 & $3.3307 \mathrm{e}-13$ & $3.3307 e-13$ \\
\hline 0.99601 & -0.99601 & 0.99601 & -0.99601 & $6.648 \mathrm{e}-13$ & $6.648 \mathrm{e}-13$ \\
\hline 0.99402 & -0.99402 & 0.99402 & -0.99402 & $9.952 \mathrm{e}-13$ & $9.952 \mathrm{e}-13$ \\
\hline 0.99203 & -0.99203 & 0.99203 & -0.99203 & $1.3243 \mathrm{e}-12$ & $1.3243 \mathrm{e}-12$ \\
\hline 0.99005 & -0.99005 & 0.99005 & -0.99005 & $1.652 \mathrm{e}-12$ & $1.652 \mathrm{e}-12$ \\
\hline 0.98807 & -0.98807 & 0.98807 & -0.98807 & $1.9785 \mathrm{e}-12$ & $1.9785 \mathrm{e}-12$ \\
\hline 0.9861 & -0.9861 & 0.9861 & -0.9861 & $2.3037 \mathrm{e}-12$ & $2.3037 \mathrm{e}-12$ \\
\hline 0.98413 & -0.98413 & 0.98413 & -0.98413 & $2.6276 \mathrm{e}-12$ & $2.6276 \mathrm{e}-12$ \\
\hline 0.98216 & -0.98216 & 0.98216 & -0.98216 & $2.9501 \mathrm{e}-12$ & $2.9501 \mathrm{e}-12$ \\
\hline 0.9802 & -0.9802 & 0.9802 & -0.9802 & $3.2715 \mathrm{e}-12$ & $3.2715 \mathrm{e}-12$ \\
\hline
\end{tabular}

الجدول (1.1) نتائج حل المسألة في المثال (2.5.1) بإستخدام طريقة رنج-كوتا الصريحة من الرتبة 


\begin{tabular}{|c|c|c|c|c|c|}
\hline \multicolumn{2}{|c|}{ قيم رنج- كوتا الصريحة من الرتبة } & \multicolumn{2}{|c|}{ القيم الحقيقية } & \multicolumn{2}{|c|}{ قيم الخطأ لرنج- كوتا الصريحة من } \\
\hline قيم & قيم & قيم & قيم & قيم الخطأ لـ & قيم الخطأ لـ \\
\hline 0.1 & -0.1 & 0.1 & -0.1 & 0 & 0 \\
\hline 0.09946 & -0.079723 & 0.099459 & -0.079707 & $5.4445 \mathrm{e}-07$ & $1.6012 \mathrm{e}-05$ \\
\hline 0.099034 & -0.063156 & 0.099032 & -0.063115 & $1.4336 \mathrm{e}-06$ & $4.1277 \mathrm{e}-05$ \\
\hline 0.098699 & -0.049621 & 0.098697 & -0.049564 & $1.9968 \mathrm{e}-06$ & $5.6747 \mathrm{e}-05$ \\
\hline 0.098437 & -0.038563 & 0.098435 & -0.038498 & $2.3244 \mathrm{e}-06$ & $6.5164 \mathrm{e}-05$ \\
\hline 0.098235 & -0.029528 & 0.098233 & -0.029459 & $2.4873 \mathrm{e}-06$ & $6.8593 \mathrm{e}-05$ \\
\hline 0.098081 & -0.022146 & 0.098079 & -0.022078 & $2.537 \mathrm{e}-06$ & $6.8578 \mathrm{e}-05$ \\
\hline 0.097966 & -0.016115 & 0.097964 & -0.016049 & $2.5105 \mathrm{e}-06$ & $6.6267 \mathrm{e}-05$ \\
\hline 0.097883 & -0.011188 & 0.09788 & -0.011125 & $2.4348 \mathrm{e}-06$ & $6.2501 \mathrm{e}-05$ \\
\hline 0.097825 & -0.0071614 & 0.097823 & -0.0071035 & $2.3292 \mathrm{e}-06$ & $5.7891 \mathrm{e}-05$ \\
\hline 0.097788 & -0.0038717 & 0.097786 & -0.0038196 & $2.1684 \mathrm{e}-06$ & $5.2121 \mathrm{e}-05$ \\
\hline
\end{tabular}

الجدول (1.2) نتائج حل المسألة في المثال (2.5.2) بإستخدام طريقة رنج-كوتا الصريحة من الرتبة الخامسة

وعليه يتضح من إستخدام طرائق رنج-كوتا الصريحة من الرتب العليا مدى كفاءة هذه

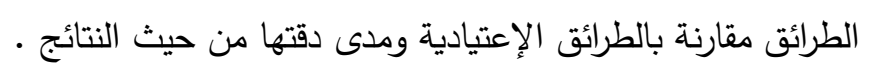

3. طرائق رنج-كوتا الضمنية من الرتب العليا:

ان أفضل الطرائق العددية لحل المسائل الصلبة هو إستخدام الطرائق الضمنية لذلك إهتم

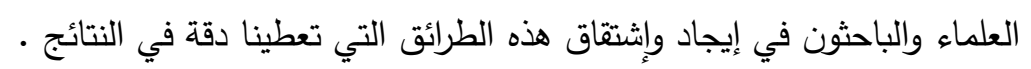

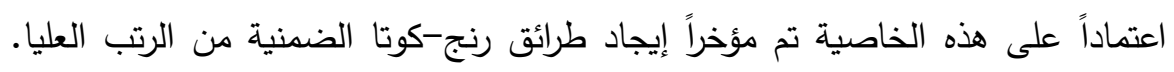

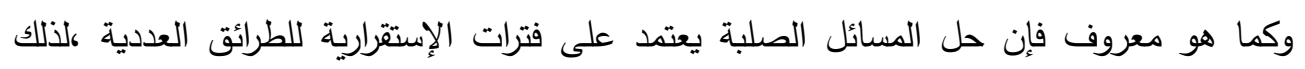

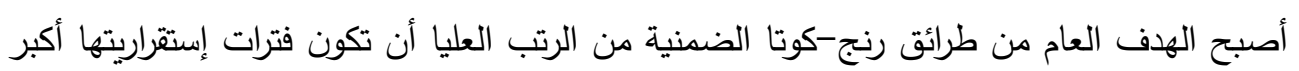

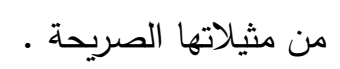

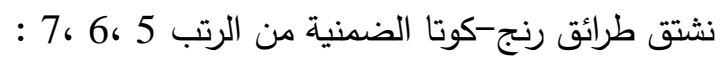


3.1 طريقة رنج-كوتا الضمنية من الرتبة الخامسة: [3]

اعتماداً على طريقة رنج-كوتا الصريحة من الرتبة الخامسة تكون صيغة رنج-كوتا

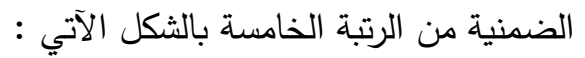

$\mathrm{y}_{\mathrm{n}}=\mathrm{y}_{\mathrm{n}+1}-\frac{\mathrm{h}}{48}\left(8 \mathrm{k}_{1}+20 \mathrm{k}_{2}+12 \mathrm{k}_{3}+6 \mathrm{k}_{4}+\mathrm{k}_{5}+\mathrm{k}_{6}\right)$

$\mathrm{k}_{1}=\mathrm{f}\left(\mathrm{x}_{\mathrm{n}+1}, \mathrm{y}_{\mathrm{n}+1}\right)$

$\mathrm{k}_{2}=\mathrm{f}\left(\mathrm{x}_{\mathrm{n}+1}-\frac{\mathrm{h}}{2}, \mathrm{y}_{\mathrm{n}+1}-\frac{1}{2} \mathrm{hk}_{1}\right)$

$\mathrm{k}_{3}=\mathrm{f}\left(\mathrm{x}_{\mathrm{n}+1}-\frac{\mathrm{h}}{2}, \mathrm{y}_{\mathrm{n}+1}-\frac{1}{2} \mathrm{hk}_{2}\right)$

$\mathrm{k}_{4}=\mathrm{f}\left(\mathrm{x}_{\mathrm{n}+1}-\mathrm{h}, \mathrm{y}_{\mathrm{n}+1}-\mathrm{hk}_{3}\right)$

$\mathrm{k}_{5}=\mathrm{f}\left(\mathrm{x}_{\mathrm{n}+1}-\mathrm{h}, \mathrm{y}_{\mathrm{n}+1}-\mathrm{hk}_{4}\right)$

$\mathrm{k}_{6}=\mathrm{f}\left(\mathrm{x}_{\mathrm{n}+1}-\mathrm{h}, \mathrm{y}_{\mathrm{n}+1}-\mathrm{hk}_{5}\right)$

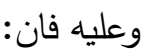

$\mathrm{y}_{\mathrm{n}+1}=\mathrm{y}_{\mathrm{n}}+\frac{\mathrm{h}}{48}\left(8 \mathrm{k}_{1}+20 \mathrm{k}_{2}+12 \mathrm{k}_{3}+6 \mathrm{k}_{4}+\mathrm{k}_{5}+\mathrm{k}_{6}\right)$

فترة إستقرارية هذه الطريقة (3.6,0-) [ انظر الثكل (2.1) ] .

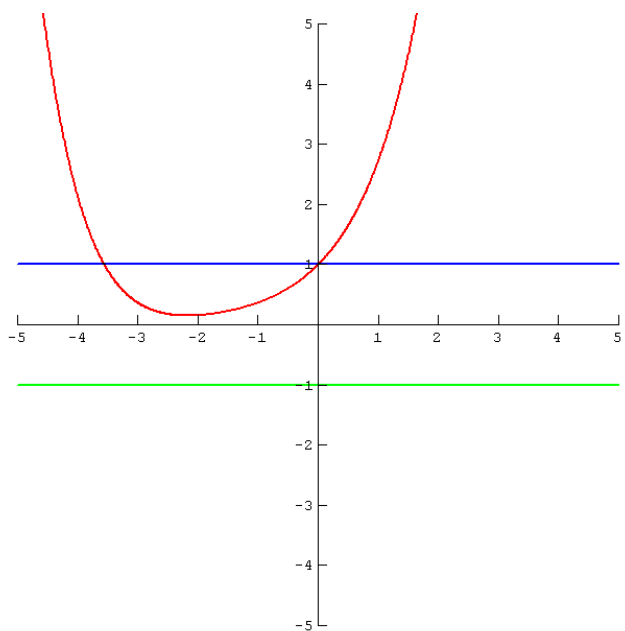

الثكل (2.1) فترة إستقرارية طريقة رنج-كوتا الضمنية من الرتبة الخامسة 


\section{2 طريقة رنج-كوتا الضمنية من الرتبة السادسة: [3]}

إعتماداً على طريقة رنج-كوتا الصريحة من الرتبة السادسة تكون صيغة رنج-كوتا لرنا

الضمنية من الرتبة السادسة بالثكل الآتي :

$y_{n}=y_{n+1}-\frac{h}{96}\left(16 k_{1}+40 k_{2}+24 k_{3}+12 k_{4}+2 k_{5}+k_{6}+k_{7}\right)$

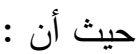

$k_{1}=f\left(x_{n+1}, y_{n+1}\right)$

$k_{2}=f\left(x_{n+1}-\frac{h}{2}, y_{n+1}-\frac{1}{2} h k_{1}\right)$

$k_{3}=f\left(x_{n+1}-\frac{h}{2}, y_{n+1}-\frac{1}{2} h k_{2}\right)$

$k_{4}=f\left(x_{n+1}-h, y_{n+1}-h k_{3}\right)$

$k_{5}=f\left(x_{n+1}-h, y_{n+1}-h k_{4}\right)$

$k_{6}=f\left(x_{n+1}-h, y_{n+1}-h k_{5}\right)$

$k_{7}=f\left(x_{n+1}-h, y_{n+1}-h k_{6}\right)$

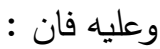

$y_{n+1}=y_{n}+\frac{h}{96}\left(16 k_{1}+40 k_{2}+24 k_{3}+12 k_{4}+2 k_{5}+k_{6}+k_{7}\right)$

فترة إستقرارية هذه الطريقة (3.7,0-) [ انظر الثكل (2.2) ] .

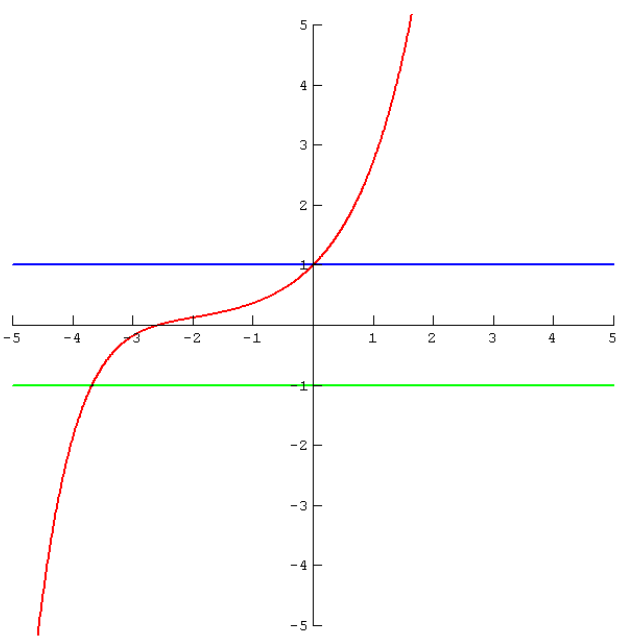

الثكل (2.2) فترة إستقرارية طريقة رنج-كوتا الضمنية من الرتبة السادسة 
3.3 طريقة رنج-كوتا الضمنية من الرتبة السابعة: [3]

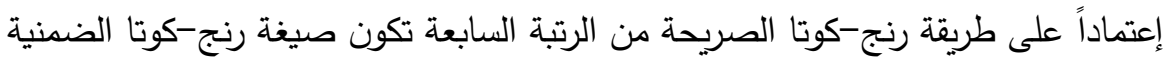
من الرتبة السابعة بالثكل الآتي:

$y_{n}=y_{n+1}-\frac{h}{192}\left(32 k_{1}+80 k_{2}+48 k_{3}+24 k_{4}+4 k_{5}+2 k_{6}+k_{7}+k_{8}\right)$

$k_{1}=f\left(x_{n+1}, y_{n+1}\right)$

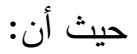

$k_{2}=f\left(x_{n+1}-\frac{h}{2}, y_{n+1}-\frac{1}{2} h k_{1}\right)$

$k_{3}=f\left(x_{n+1}-\frac{h}{2}, y_{n+1}-\frac{1}{2} h k_{2}\right)$

$k_{4}=f\left(x_{n+1}-h, y_{n+1}-h k_{3}\right)$

$k_{5}=f\left(x_{n+1}-h, y_{n+1}-h k_{4}\right)$

$k_{6}=f\left(x_{n+1}-h, y_{n+1}-h k_{5}\right)$

$k_{7}=f\left(x_{n+1}-h, y_{n+1}-h k_{6}\right)$

$k_{8}=f\left(x_{n+1}-h, y_{n+1}-h k_{7}\right)$

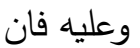

$y_{n+1}=y_{n}+\frac{h}{192}\left(32 k_{1}+80 k_{2}+48 k_{3}+24 k_{4}+4 k_{5}+2 k_{6}+k_{7}+k_{8}\right)$

فترة إستقرارية هذه الطريقة (4.7,0) [ انظر الثكل(2.3) ] •

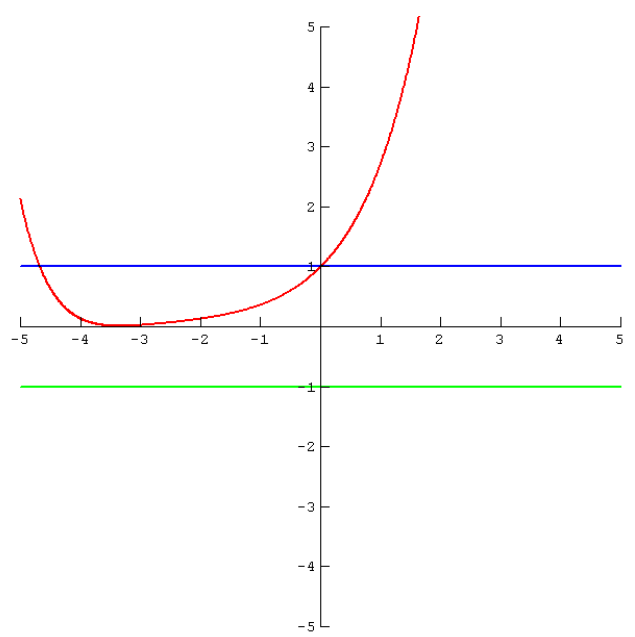

الثكل (2.3) فترة إستقرارية طريقة رنج-كوتا الضمنية من الرتبة السابعة 


\section{4}

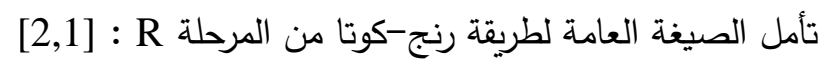

$\mathrm{y}_{\mathrm{n}+1}=\mathrm{y}_{\mathrm{n}}+\mathrm{h} \phi\left(\mathrm{x}_{\mathrm{n}+1}, \mathrm{y}_{\mathrm{n}+1}, \mathrm{~h}\right)$

يقال بأن صيغة رنج-كوتا الضمنية (7) من المرحلة R هي من الرتبة P إذا كان : [2,1] $\mathrm{y}\left(\mathrm{x}_{\mathrm{n}+1}\right)-\mathrm{y}_{\mathrm{n}+1}=\mathrm{O}\left(\mathrm{h}^{\mathrm{p}+1}\right)$

بإستخدام الصيغة (7) على معادلة الفروقات نحصل على : [2,1]

$\mathrm{y}_{\mathrm{n}+1}=\mathrm{E}(\lambda \mathrm{h}) \mathrm{y}_{\mathrm{n}}$

$\mathrm{y}^{\prime}=\lambda \mathrm{y}, \mathrm{y}\left(\mathrm{x}_{0}\right)=\mathrm{y}_{0}$

وبإستخدام مسألة الإختبار : [2,1]

نحصل على : [2,1]

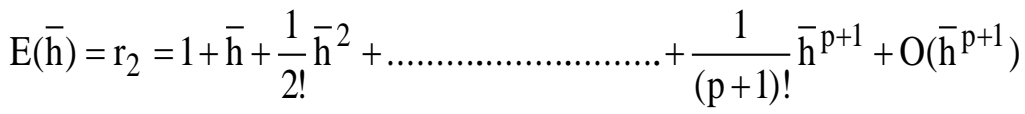

حيث وعليه تكون فترات الإستقرارية المطلقة لطرائق رنج-كوتا الضمنية من الرتب العليا كما في

الجدول الآتي • مع الأخذ بنظر الإعتيار أنه تم إيجاد هذه الفترات بإستخدام الرسم الآتي :

\begin{tabular}{|c|c|c|}
\hline RK & $r_{2}$ & فترة الإستقرارية| \\
\hline 5 & $1+\bar{h}+\frac{\bar{h}^{2}}{2 !}+\frac{\bar{h}^{3}}{3 !}+\frac{\bar{h}^{4}}{4 !}+\frac{\bar{h}^{5}}{5 !}+\frac{\bar{h}^{6}}{6 !}$ & $(-3.6,0)$ \\
\hline 6 & $1+\bar{h}+\frac{\bar{h}^{2}}{2 !}+\frac{\bar{h}^{3}}{3 !}+\frac{\bar{h}^{4}}{4 !}+\frac{\bar{h}^{5}}{5 !}+\frac{\bar{h}^{6}}{6 !}+\frac{\bar{h}^{7}}{7 !}$ & $(-3.7,0)$ \\
\hline 7 & $1+\bar{h}+\frac{\bar{h}^{2}}{2 !}+\frac{\bar{h}^{3}}{3 !}+\frac{\bar{h}^{4}}{4 !}+\frac{\bar{h}^{5}}{5 !}+\frac{\bar{h}^{6}}{6 !}+\frac{\bar{h}^{7}}{7 !}+\frac{\bar{h}^{8}}{8 !}$ & $(-4.7,0)$ \\
\hline
\end{tabular}

3.5 تطبيقات على المسائل الصلبة:

في هذه الفقرة سوف يتم تطبيق طرائق رنج-كوتا الضمنية من الرتب العليا على المسائل الصلبة مع بيان مدى كفاءة هذه الطرائق . لـون 
مثال (3.5.1) : (3)

حل نظام المعادلات في المثال (2,5,1) بإستخدام طريقة رنج-كوتا الضمنية من الرتبة

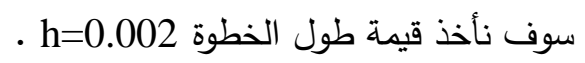

h تشير طرائق رنج-كوتا الضمنية من الرتب العليا إلى مدى تأثير إستخدام طول الخطوة

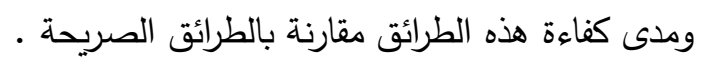

مثال (3.5.2) :

حل نظام المعادلات في المثال (2,5,2) بإستخدام طريقة رنج-كوتا الضمنية من الرتبة

الخامسة .

سوف نأخذ قيمة طول الخطوة h=0.001 .

وعليه يتضح من إستخدام طرائق رنج-كوتا الضمنية من الرتب العليا مدى كفاءة هذه

الطرائق مقارنة بالطرائق الصريحة ومدى دقتها في النتائج •

\begin{tabular}{|c|c|c|c|c|c|}
\hline \multicolumn{2}{|c|}{ قيم رنج- كوتا الضمنية من الرتبة } & \multicolumn{2}{|c|}{ القيم الحقيقية } & \multicolumn{2}{|c|}{ قيم الخطأ لرنج- كوتا الضمنية من } \\
\hline$y_{1}$ & قيم & قيم $y_{1}$ & قيم & قيم الخطأ لـ $y_{1}$ & قيم الخطأ لـ $y_{2}$ \\
\hline 1 & -1 & 1 & -1 & 0 & 0 \\
\hline 0.998 & -0.998 & 0.998 & -0.998 & $3.3362 \mathrm{e}-13$ & $3.3351 \mathrm{e}-13$ \\
\hline 0.99601 & -0.99601 & 0.99601 & -0.99601 & $6.6713 e-13$ & $6.6702 \mathrm{e}-13$ \\
\hline 0.99402 & -0.99402 & 0.99402 & -0.99402 & $1.0006 \mathrm{e}-12$ & $1.0003 \mathrm{e}-12$ \\
\hline 0.99203 & -0.99203 & 0.99203 & -0.99203 & $1.3343 \mathrm{e}-12$ & $1.3335 \mathrm{e}-12$ \\
\hline 0.99005 & -0.99005 & 0.99005 & -0.99005 & $1.6679 \mathrm{e}-12$ & $1.6678 \mathrm{e}-12$ \\
\hline 0.98807 & -0.98807 & 0.98807 & -0.98807 & $2.0014 \mathrm{e}-12$ & $2.0012 \mathrm{e}-12$ \\
\hline 0.9861 & -0.9861 & 0.9861 & -0.9861 & $2.3349 \mathrm{e}-12$ & $2.3354 \mathrm{e}-12$ \\
\hline 0.98413 & -0.98413 & 0.98413 & -0.98413 & $2.6685 \mathrm{e}-12$ & $2.6686 \mathrm{e}-12$ \\
\hline 0.98216 & -0.98216 & 0.98216 & -0.98216 & $3.002 \mathrm{e}-12$ & $3.0024 \mathrm{e}-12$ \\
\hline 0.9802 & -0.9802 & 0.9802 & -0.9802 & $3.3354 \mathrm{e}-12$ & $3.3356 \mathrm{e}-12$ \\
\hline
\end{tabular}

الجدول (2.1) نتائج حل المسألة في المثال (3.5.1) بإستخدام طريقة رنج-كوتا الضمنية من الرتبة الخامسة 


\begin{tabular}{|c|c|c|c|c|c|}
\hline \multicolumn{2}{|c|}{ قيم رنج- كوتا الضمنية من الرتبة } & \multicolumn{2}{|c|}{ القيم الحقيقية } & \multicolumn{2}{|c|}{ قيم الخطأ لرنج- كوتا الضمنية من } \\
\hline قيم & قيم & قيم & قيم & قيم الخطأ & قيم الخطأ \\
\hline 0.1 & -0.1 & 0.1 & -0.1 & 0 & 0 \\
\hline 0.09946 & -0.079715 & 0.099459 & -0.079707 & $2.8755 \mathrm{e}-07$ & $8.1918 \mathrm{e}-06$ \\
\hline 0.099033 & -0.063142 & 0.099032 & -0.063115 & $9.7337 \mathrm{e}-07$ & $2.7068 \mathrm{e}-05$ \\
\hline 0.098698 & -0.049602 & 0.098697 & -0.049564 & $1.3745 \mathrm{e}-06$ & $3.732 \mathrm{e}-05$ \\
\hline 0.098437 & -0.038539 & 0.098435 & -0.038498 & $1.5724 \mathrm{e}-06$ & $4.1473 \mathrm{e}-05$ \\
\hline 0.098234 & -0.029501 & 0.098233 & -0.029459 & $1.6311 \mathrm{e}-06$ & $4.1419 \mathrm{e}-05$ \\
\hline 0.09808 & -0.022116 & 0.098079 & -0.022078 & $1.5966 \mathrm{e}-06$ & $3.8559 \mathrm{e}-05$ \\
\hline 0.097965 & -0.016083 & 0.097964 & -0.016049 & $1.5021 \mathrm{e}-06$ & $3.3923 \mathrm{e}-05$ \\
\hline 0.097882 & -0.011153 & 0.09788 & -0.011125 & $1.3713 \mathrm{e}-06$ & $2.8257 \mathrm{e}-05$ \\
\hline 0.097824 & -0.0071256 & 0.097823 & -0.0071035 & $1.221 \mathrm{e}-06$ & $2.2095 \mathrm{e}-05$ \\
\hline 0.097787 & -0.0038347 & 0.097786 & -0.0038196 & $1.0238 \mathrm{e}-06$ & $1.5057 \mathrm{e}-05$ \\
\hline
\end{tabular}

الجدول (2.2) نتائج حل المسألة في المثال (3.5.2) بإستخدام طريقة رنج-كوتا الضمنية من الرتبة الخامسة

: إإستتتاجات

إن أهم ماتتاوله البحث هو طرائق رنج-كوتا من الرتب العليا لحل مسائل القيم الإبتدائية الصلبة التي تعد من الأمور المهمة لتعلقها بموضوعات مهمة في حياتتا اليومية مثل موضوعات الكيمياء وشبكة المعلومات وغيرهما مما يدخل فيه هذا النوع من المسائل . تم إثتقاق طرائق رنج-كوتا الصريحة من الرتب العليا لحل المسائل الصلبة، وتم إستخدام هذه الطرائق لحل المسائل الصلبة وقد تبين أن هذه الطرائق ناجحة في المسائل الصلبة إذ تمباديا الحصول على نتائج وكانت دقيقة . كما تم إثتقاق طرائق رنج-كوتا الضمنية من الرتب العليا لحل المسائل الصلبة وقد كانت هذه الطرائق أفضل من مثيلاتها الصريحة في حل هذا النوع من المسائل وتم الحصول على نلى نتائج

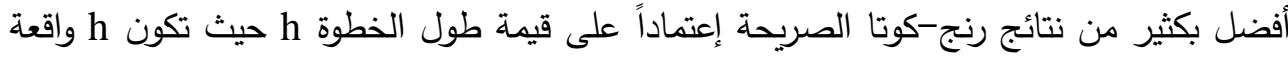
داخل منطقة الإستقرارية لطرائق رنج-كوتا من الرتب العليا بنوعيها الصريحة والضمنية .

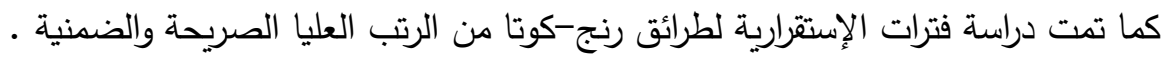




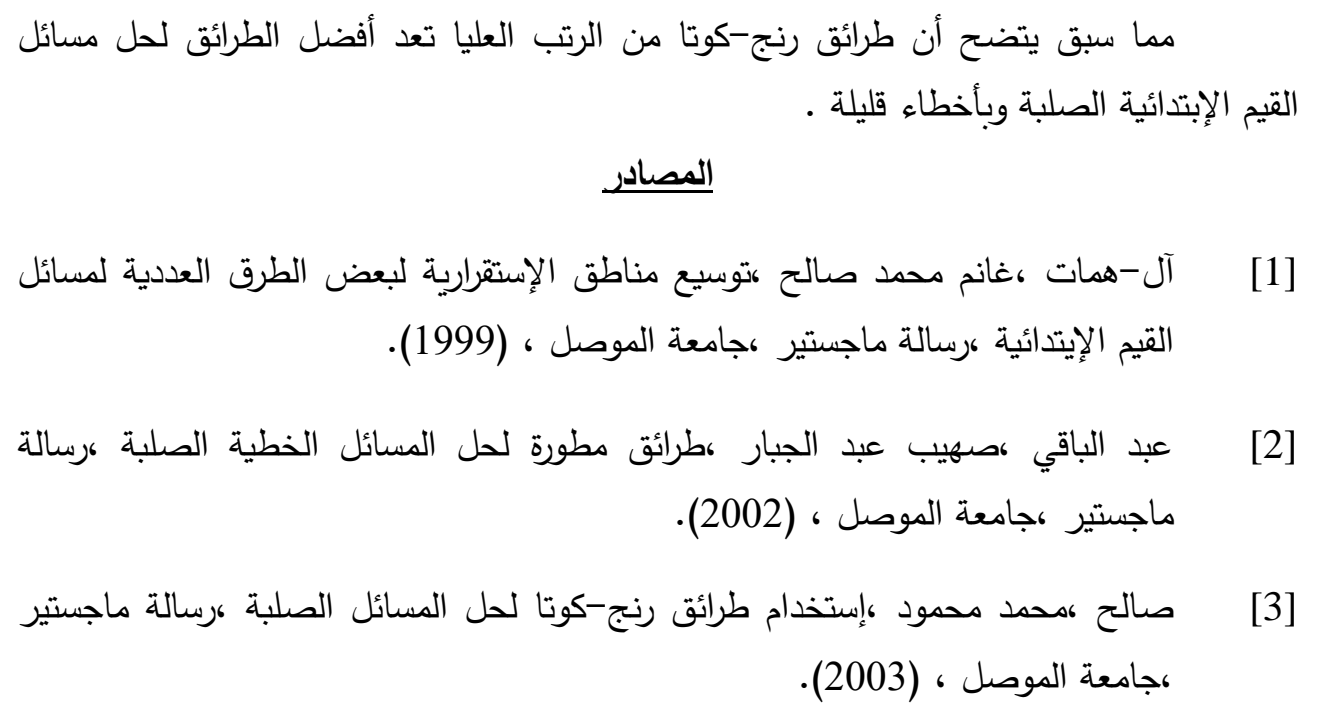

[4] Butcher, J.C. and Diamantakis, M.T., DESIRE: Diagonally extended singly implicit Runge-Kutta effective order methods, Numeric. Algorithm, 17, (1998), pp. 121-145.

[5] Butcher, J.C., Numerical methods for diff. eq.s and applications, the Arabian Journal for Science and Engineering, Dharan, Saudi Arabia, 22(2Command), (1997), pp. 17-29.

[6] Cash, J. R., Block Runge-Kutta methods for numerical integration of initial value problems in ordinary diff. eq.s Part II: the stiff case, Math. Of Computation, Vol. 40, No. 161, (1983), pp. 193-206.

[7] Cash, J. R., A Class of implicit Runge-Kutta methods for the numerical integration of stiff ordinary diff. eq.s, Journal of the Association of Computing Machinery, Vol. 22, No. 4, (1975), pp. 504-511.

[8] Cash, J.R., Runge-Kutta methods for the solution of stiff two-point boundary value problems, Applied Numeric. Math., Vol. 22, (1996), pp. 165-177.

[9] Murshed, Abdul-Habib Abdullah, New Parallel numerical algorithms for solving stiff ordinary diff. eq.s adapted for MIMD Computers, Ph.D. thesis, Univ. of Mosul, (2000).

[10] Roche, M., Lubich, C. and Hairer, E., Error of Rosenbrock method for stiff ordinary diff. eq.s, Bit, 29, (1989), pp. 77-90. 
[11] Voss, D.A. and Casper M.J., Efficient split linear multistep methods for stiff ordinary diff. eq.s, SIAM Journal Sci. Stat. Comput., Vol. 19, No. 5, (1989), pp. 990-999.

[12] Voss, D.A., Factored two-step Runge-Kutta methods, App. Math. And Comput., Vol. 31, (1989), pp. 361-368.

[13] Voss, D.A., Fifth-order exponentially fitted formula, SIAM Journal Numeric. Anal., Vol. 25, No. 3, (1988), pp. 670-678. 